\title{
THE LAW OF THE ITERATED LOGARITHM FOR LACUNARY TRIGONOMETRIC SERIES
}

\author{
BY \\ MARY WEISS( $\left.{ }^{1}\right)$
}

1. The purpose of this paper is to prove the following theorem of "The Law of the Iterated Logarithm" for lacunary trigonometric series.

THEOREM 1. Let

$$
S(x)=\sum_{k=0}^{\infty}\left(a_{k} \cos n_{k} x+b_{k} \sin n_{k} x\right)
$$

be a lacunary trigonometric series, that is to say, one such that $n_{k+1} / n_{k}>q>1$ for all $k$. We write

$$
\begin{aligned}
B_{N} & =\left(\frac{1}{2} \sum_{k=1}^{N}\left(a_{k}^{2}+b_{k}^{2}\right)\right)^{1 / 2}, \quad M_{N}=\operatorname{Max}_{1 \leqq k \leqq N}\left(a_{k}^{2}+b_{k}^{2}\right)^{1 / 2}, \\
S_{N}(x) & =\sum_{k=1}^{N}\left(a_{k} \cos n_{k} x+b_{k} \sin n_{k} x\right) .
\end{aligned}
$$

If, for $N \rightarrow+\infty$,

$$
B_{N} \rightarrow \infty \quad \text { and } \quad M_{N}=o\left\{\frac{B_{N}}{\left(\log \log B_{N}\right)^{1 / 2}}\right\}
$$

then we have, for almost all $x$,

$$
\limsup _{N \rightarrow+\infty} \frac{S_{N}(x)}{\left(2 B_{N}^{2} \log \log B_{N}\right)^{1 / 2}}=1
$$

In this theorem the $a_{k}$ and $b_{k}$ are real and the $n_{k}$ are positive but not necessarily integral. The latter point is important for applications.

The Law of the Iterated Logarithm for lacunary series has already been treated in the literature. In the first place, Salem and Zygmund [1] (numbers in square brackets refer to the bibliography at the end of the paper) have shown that under the hypotheses (1.2) we have (1.3) with "ฏ” instead of "=." They also presuppose that the $n_{k}$ are integers. A complete proof of (1.3) was given later by Erdös and Gál [1] under the restriction however

Presented to the Society February 25, 1956; received by the editors August 19, 1957.

(1) The research resulting in this paper was supported by the Office of Scientific Research of the Air Force under contract AF18(600)-1111. The paper is part of a Ph.D. thesis submitted to the Department of Mathematics of the University of Chicago. 
that $S$ is of the form $\sum e^{i n_{k} x}$ and that the $n_{k}$ are integers. In the proof which follows we use some ideas from these two papers and also from the classical paper of Kolmogoroff [1] on the Law of the Iterated Logarithm for independent random variables. More detailed acknowledgments will be given at the proper places.

I am grateful to Professor Zygmund for calling my attention to the problem and for helping me with suggestions.

2. From now on, we shall assume for simplicity of writing that (1.1) is a cosine series:

$$
\sum_{1}^{\infty} a_{k} \cos n_{k} x
$$

The proof is the same in the general case as we can see by writing $a_{k} \cos n_{k} x$ $+b_{k} \sin n_{k} x=\rho_{k} \cos \left(n_{k} x-\phi_{k}\right)$.

When no ambiguity can arise, we write $B, M$, and $S$ instead of $B_{N}, M_{N}$, and $S_{N}$.

We will prove Theorem 1 in the following form:

THEOREM 1'. If $\epsilon>0, \delta>0$ are given (small) quantities, and $N$ is a given integer, then under the hypotheses (1.2), there exists a finite sequence of integers, $N_{0}<N_{1} \cdots<N_{k}$ such that

$$
\operatorname{Max}_{1 \leqq j \leqq k} \frac{S_{N_{j}}(x)}{\left(2 B_{N j}^{2} \log \log B_{N_{j}}\right)^{1 / 2}} \geqq 1-\epsilon
$$

for $x$ in a set of measure greater than $2 \pi-\delta$.

3. We first prove a lemma about lacunary series satisfying certain special conditions.

Definition. We say that a lacunary series satisfies condition $(r, R)$ if it consists of blocks of terms of length between $R / 3$ and $R$, separated by empty blocks of length between $r / 3$ and $r$.

LemMa 1. Let

$$
S(x)=\sum a_{k} \cos n_{k} x
$$

be a finite series satisfying condition $(r, R)$ where

$$
\left(q^{(1 / 3) r}-1\right)^{-1} \leqq(1 / 4)(q-1)
$$

and

$$
q^{-(1 / 3) R}(R+1)^{2} \leqq 1 / 2 .
$$

Let $\lambda, \eta$ be positive numbers and $(\alpha, \beta)$ a fixed finite interval. Let

$$
M=\max \left|a_{k}\right|, \quad B^{2}=\frac{1}{2} \sum a_{k}^{2} .
$$


Then if

$$
n_{1}^{-1} \lambda M R^{2} A_{q} \leqq \frac{1}{2}(\beta-\alpha)
$$

and

$$
A \lambda M R^{2} \leqq \frac{1}{4} \eta
$$

where $A$ is a certain absolute constant and $A_{q}$ a certain constant depending only on $q$, we have

$$
\frac{1}{2}(\beta-\alpha) e^{(1-\eta) \lambda^{2} B^{2} / 2} \leqq \int_{\alpha}^{\beta} e^{\lambda S} d x \leqq \frac{3}{2}(\beta-\alpha) e^{(1+\eta) \lambda^{2} B^{2} / 2} .
$$

The proof of the above is similar to a proof in the Salem-Zygmund paper of a lemma similar to this lemma but having as a conclusion,

$$
\int_{0}^{2 \pi} e^{\lambda S} d x \leqq 2 \pi e^{(1+\eta) \lambda^{2} B^{2} / 2}
$$

instead of (3.5).

In what follows, $A$ will be an absolute constant and $A_{q}$ a constant depending only on $q$, not necessarily the same at each appearance.

The proof is based on the inequality.

$$
\left|\log \left(1+z+\frac{1}{2} z^{2}\right)-z\right| \leqq A|z|^{3}
$$

valid for, say, $|z| \leqq 1 / 2$. We define

$$
\Delta_{h}=\sum a_{k} \cos n_{k} x
$$

for $a_{k} \cos n_{k} x$ in the $h$ th nonempty block of $S$, and

$$
\delta_{h}=\frac{1}{2} \sum a_{k}^{2}
$$

where the summation is taken over coefficients $a_{k}$ belonging to the $h$ th nonempty block of $S$.

Now if $\lambda M R<1 / 2$, then $\lambda \Delta_{h} \leqq 1 / 2$ for all $h$, and (3.6) implies

$$
e^{\lambda \Delta_{h}}=\left(1+\lambda \Delta_{h}+\frac{1}{2} \lambda^{2} \Delta_{h}^{2}\right) e^{Q_{h}}
$$

where

$$
\left|Q_{h}\right| \leqq A \lambda^{3}\left|\Delta_{h}\right|^{3} \leqq A \lambda^{3} R^{2} \sum\left|a_{k}\right|^{3} \leqq A \lambda^{3} R^{2} M \delta_{h}
$$


If, therefore, we set

$$
w_{h}=1+\lambda \Delta_{h}+\frac{1}{2} \lambda^{2} \Delta_{h}^{2}=1+\frac{1}{2} \lambda^{2} \delta_{h}+\lambda \Delta_{h}+\frac{1}{2} \lambda^{2} \Delta_{h}^{*}
$$

(so that $\Delta_{h}^{*}$ is the variable part of $\Delta_{h}^{2}$ ), we obtain

$$
\begin{aligned}
\exp \left(-A \lambda^{3} R^{2} M\right. & \left.\left.\sum \delta_{h}\right) \int_{\alpha}^{\beta}\left(\Pi w_{h}\right)\right) d x \\
\leqq & \int_{\alpha}^{\beta} e^{\lambda S} d x \leqq \exp \left(\left(A \lambda^{3} R^{2} M \sum \delta_{h}\right) \int_{\alpha}^{\beta}\left(\prod w_{h}\right) d x .\right.
\end{aligned}
$$

Hence, in order to estimate $\int_{\alpha}^{\beta} e^{\lambda S} d x$ from above and below, we need only estimate $\int_{\alpha}^{\beta}\left(\Pi w_{h}\right) d x$. Clearly,

$$
\begin{aligned}
\int_{\alpha}^{\beta}\left(\Pi w_{h}\right) d x= & (\beta-\alpha) \Pi\left(1+\frac{1}{2} \lambda^{2} \delta_{h}\right) \\
& +\int_{\alpha}^{\beta}\left[\Pi w_{h}-\Pi\left(1+\frac{1}{2} \lambda^{2} \delta_{h}\right)\right] d x .
\end{aligned}
$$

We consider the second, or "error" term. The constant term of the trigonometric polynomial $w_{h}$ is $1+2^{-1} \lambda^{2} \delta_{h}$. The number of nonconstant terms of $w_{h}$ is less than or equal to $R^{2}+R$. Now we consider a term of $\prod w_{h}-\prod\left(1+2^{-1} \lambda^{2} \delta_{h}\right)$ whose highest non constant factor is from $w_{p}$. The number of such terms is less than or equal to $\left(R^{2}+R+1\right)^{p} \leqq(R+1)^{2 p}$. In order to determine the integral over $(\alpha, \beta)$ of such a term we must first determine its order. If $\tilde{n}_{h}$ denotes the smallest of the orders of the cosines in $\Delta_{h}$ and $\bar{n}_{h}$ the largest, then the smallest of the orders of the cosines in $w_{h}$ is greater than $(q-1) \tilde{n}_{h}$ assuming, as we may, that $1<q \leqq 2$, otherwise the lowest order is $\tilde{n}_{h}$; the largest of the orders of the cosines of $w_{h}$ is $2 \bar{n}_{h}$. If $n_{k_{j}}$ denotes the order of the cosine factor of our term taken from $w_{j}$, then the order of our term is greater than or equal to

$$
\begin{aligned}
n_{k_{p}}-n_{k_{p-1}}-\cdots-n_{k_{1}} & \geqq \tilde{n}_{p}\left(q-1-2 q^{-r / 3}-2\left(q^{-r / 3}\right)^{2}-\cdots-2\left(q^{-r / 3}\right)^{p-1}\right) \\
& \geqq \tilde{n}_{p}\left(q-1-2\left(q^{r / 3}-1\right)^{-1}\right) \geqq \frac{1}{2} \tilde{n}_{p}(q-1) .
\end{aligned}
$$

The last inequality follows from (3.1).

Since the integral of $\cos n x$ over any interval is numerically less than or equal to $2 / n$, the integral of our term over $(\alpha, \beta)$ is less than or equal to

$$
\tilde{n}_{v}^{-1} A_{q} \lambda M \prod\left(1+\frac{1}{2} \lambda^{2} \delta_{h}\right) \text {. }
$$

Now 


$$
\tilde{n}_{p}^{-1} \leqq n_{1}^{-1}\left(q^{-R / 3}\right)^{p-1} .
$$

Thus the contribution to $\int_{\alpha}^{\beta}\left(\prod w_{h}-\Pi\left(1+\lambda^{2} \delta_{h}\right) / 2\right) d x$ from those terms of the integrand whose highest non constant factor comes from $w_{p}$ is less than or equal to

$$
\frac{A_{q}(R+1)^{2 p} \lambda M}{n_{1}\left(q^{R / 3}\right)^{p-1}} \Pi\left(1+\frac{1}{2} \lambda^{2} \delta_{h}\right) .
$$

Summing (3.11) over all $p$ and using (3.2) and (3.3) we obtain

$$
\begin{aligned}
\int_{\alpha}^{\beta}\left(\Pi w_{h}-\Pi\left(1+\frac{1}{2} \lambda^{2} \delta_{h}\right)\right) d x & \leqq n_{1}^{-1} \lambda M A_{q} R^{2} \Pi\left(1+\frac{1}{2} \lambda^{2} \delta_{h}\right) \\
& \leqq \frac{1}{2} \Pi\left(1+\frac{1}{2} \lambda^{2} \delta_{h}\right)(\beta-\alpha)
\end{aligned}
$$

and hence (see equation (3.9))

$$
\frac{1}{2}(\beta-\alpha) \Pi\left(1+\frac{1}{2} \lambda^{2} \delta_{h}\right)
$$

$$
\leqq \int_{\alpha}^{\beta} \Pi w_{h} d x \leqq \frac{3}{2}(\beta-\alpha) \Pi\left(1+\frac{1}{2} \lambda^{2} \delta_{h}\right) .
$$

We note that if $u$ is small, $e^{u(1-u)} \leqq 1+u \leqq e^{u}$, and hence

$$
\begin{aligned}
\exp \left(\sum \frac{1}{2} \lambda^{2} \delta_{h}-\sum \frac{1}{4} \lambda^{4} \delta_{h}^{2}\right) & \leqq \prod\left(1+\frac{1}{2} \lambda^{2} \delta_{h}\right) \\
& \leqq \exp \left(\sum \frac{1}{2} \lambda^{2} \delta_{h}\right)
\end{aligned}
$$

Now

$$
\sum \frac{1}{4} \lambda^{4} \delta_{h}^{2} \leqq \frac{1}{8} \lambda^{4} M^{2} R \sum a_{k}^{2} \leqq \frac{1}{4} \lambda^{2} M^{2} R \lambda^{2} B^{2} .
$$

Since by (3.4) we have $\lambda^{2} M^{2} R^{2} \leqq \eta$, equation (3.15) gives us

$$
\sum \frac{1}{4} \lambda^{4} \delta_{h}^{2} \leqq \frac{1}{4} \eta \lambda^{2} B^{2}
$$

Hence, (3.14) becomes

$$
\exp \left(\frac{1}{2} \lambda^{2} B^{2}\left(1-\frac{1}{2} \eta\right)\right) \leqq \prod\left(1+\frac{1}{2} \lambda^{2} \delta_{h}\right) \leqq \exp \left(\frac{1}{2} \lambda^{2} B^{2}\right) .
$$

We can see from inspection of (3.8) that in order to obtain (3.5) we have only 
to estimate $A \lambda^{3} R^{2} M \sum \delta_{h}=A \lambda^{3} R^{2} M B^{2}$. By (3.4), $A \lambda M R^{2} \leqq \eta / 4$, and hence

$$
A \lambda^{3} R^{2} M B^{2} \leqq \frac{1}{4} \eta \lambda^{2} B^{2}
$$

Equations (3.16), (3.17), and (3.8) give us

$$
\frac{1}{2}(\beta-\alpha) e^{(1-\eta) \lambda^{2} B^{2} / 2} \leqq \int_{\alpha}^{\beta} e^{\lambda S} d x \leqq \frac{3}{2}(\beta-\alpha) e^{(1+\eta) \lambda^{2} B^{2} / 2}
$$

which is what we wished to prove.

4. We denote by $W_{\alpha, \beta}(y)$ the measure of the set of points $x$ in $(\alpha, \beta)$ for which $S(x) \geqq y$. That is

$$
W_{\alpha, \beta}(y)=|\{x: S(x) \geqq y ; x \in(\alpha, \beta)\}| .
$$

We will drop the subscripts $\alpha, \beta$ where they are understood.

We now use Lemma 1 to obtain estimates for $W_{\alpha, \beta}(y)$.

Lemma 2. Let $S$ be a finite series satisfying condition $(r, R)$ and in addition the hypotheses (3.1) and (3.2) of Lemma 1. Given $\epsilon>0$, let $\delta, \epsilon^{\prime}$ and $\eta$ be defined by the following equations:

(4.1) $\quad\left(1-\epsilon^{\prime}\right)(1+4 \delta)=1 ; \quad\left(1-\epsilon^{\prime}\right)(1-\delta)=1-\epsilon ; \quad \eta=\frac{1}{8} \delta^{2}$.

Then, if for

$$
\lambda=\frac{20}{B}(2 \log \log B)^{1 / 2}
$$

the hypotheses (3.3) and (3.4) of Lemma 1 are satisfied, and if $B$ is larger than some fixed $B_{0}$ depending only on $\delta$, we have

$$
W\left((1-\epsilon)\left(2 B^{2} \log \log B\right)^{1 / 2}\right) \geqq \frac{(\beta-\alpha)}{\log B} .
$$

This argument follows closely that of a similar lemma in the paper of Kolmogoroff [1]. Let

$$
\begin{gathered}
a=\frac{\left(1-\epsilon^{\prime}\right)}{B}(2 \log \log B)^{1 / 2} . \\
\int_{\alpha}^{\beta} e^{a S} d x=-\int_{-\infty}^{\infty} e^{a y} d W(y)=a \int_{-\infty}^{\infty} e^{a y} W(y) d y \\
=a\left(\int_{-\infty}^{0}+\int_{0}^{a B^{2}(1-\delta)}+\int_{a B^{2}(1-\delta)}^{a B^{2}(1+\delta)}+\int_{a B^{2}(1+\delta)}^{8 a B^{2}}+\int_{8 a B^{2}}^{+\infty}\right) \\
=a\left(J_{1}+J_{2}+J_{3}+J_{4}+J_{5}\right) .
\end{gathered}
$$


Estimation of $J_{1}$ :

$$
a J_{1}=a \int_{-\infty}^{0} e^{a y} W(y) d y \leqq a \int_{-\infty}^{0} e^{a y}(\beta-\alpha) d y \leqq(\beta-\alpha) .
$$

Estimation of $J_{5}$ :

Letting $\lambda=8 a$ and applying Lemma 1 , we have

$$
\begin{aligned}
W(y) & \leqq \frac{3}{2}(\beta-\alpha) \exp \left(\frac{1}{2}(1+\eta) \lambda^{2} B^{2}-\lambda y\right) \\
& \leqq \frac{3}{2}(\beta-\alpha) \exp \left(\frac{1}{2}(1+\eta) 64 a^{2} B^{2}-8 a y\right) .
\end{aligned}
$$

If $y \geqq 8 a B^{2}$, then

$$
\frac{1}{2}(1+\eta) 64 a^{2} B^{2}-8 a y \leqq \frac{1}{2} 8 a y(1+\eta)-8 a y \leqq-2 a y .
$$

Therefore,

$$
W(y) \leqq \frac{3}{2}(\beta-\alpha) e^{-2 a y}
$$

and hence

$$
a J_{5}=a \int_{8 a B^{2}}^{\infty} e^{a y} W(y) d y \leqq \frac{3}{2} a(\beta-\alpha) \int_{8 a B^{2}}^{\infty} e^{-a y} d y \leqq \frac{3}{2}(\beta-\alpha) e^{-8 a^{2} B^{2}} .
$$

Estimation of $J_{2}$ and $J_{4}$ :

Let $\lambda=y \mid B^{2}$; since in $J_{2}$ and $J_{4}, y \leqq 8 a B^{2} \leqq 8 B(2 \log \log B)^{1 / 2}$, we have $\lambda \leqq 8 B^{-1}(2 \log \log B)^{1 / 2}$ which allows us to apply Lemma 1 . Thus

$$
W(y) \leqq \frac{3}{2}(\beta-\alpha) e^{-(1-\eta) y^{2} B^{-2} / 2}=\frac{3}{2}(\beta-\alpha) e^{-\left(1-\delta^{2} / 8\right) y^{2} B^{-2} / 2}
$$

and

$$
a\left(J_{2}+J_{4}\right) \leqq a\left(\int_{0}^{a B^{2}(1-\delta)}+\int_{a B^{2}(1+\delta)}^{8 a B^{2}}\right)\left(e^{-a y-\left(1-\delta^{2} / 8\right) y^{2} B^{-2} / 2} \cdot \frac{3}{2}(\beta-\alpha)\right) .
$$

The maximum of the function $a y-\left(1-\delta^{2} / 8\right) y^{2} B^{-2} / 2$ is located at the point $y=a B^{2}\left(1-\delta^{2} / 8\right)^{-1}$. Hence in the interval

$$
\left(a B^{2}(1+\delta), 8 a B^{2}\right), \quad a y-\left(1-\delta^{2} / 8\right)\left(y^{2} B^{-2}\right) / 2
$$

is majorized by its value at $y=a B^{2}(1+\delta)$ which is

$$
a B^{2}(1+\delta)-\left(1-\delta^{2} / 8\right)(1+\delta)^{2} a^{2} B^{2} / 2 \leqq\left(1-\delta^{2} / 2\right) a^{2} B^{2} / 2 .
$$


Similarly, in the interval $\left(0, a B^{2}(1-\delta)\right)$ we have $a y-\left(1-\delta^{2} / 8\right) y^{2} B^{-2} / 2$ $\leqq\left(1-\delta^{2} / 2\right) a^{2} B^{2} / 2$. Hence

$$
a\left(J_{2}+J_{4}\right) \leqq 12(\beta-\alpha) a^{2} B^{2} e^{\left(1-\delta^{2} / 2\right) a^{2} B^{2} / 2} .
$$

If $B$ is so large that $12 a^{2} B^{2} \leqq 3\left(e^{a^{2} B^{2} \delta^{2} / 8}\right) / 16$, the above equation gives us, together with Lemma 1

$$
a\left(J_{2}+J_{4}\right) \leqq \frac{3}{16}(\beta-\alpha) e^{\left(1-\delta^{2} / 4\right) a^{2} B^{2} / 2} \leqq \frac{1}{2} \int_{\alpha}^{\beta} e^{a S} d x .
$$

For $B$ so large that $15(\beta-\alpha) \leqq e^{(1-\eta) a^{2} B^{2} / 2}$, we have, by Lemma 1 ,

$$
a\left(J_{1}+J_{5}\right) \leqq \frac{5}{2}(\beta-\alpha) \leqq \frac{1}{6} e^{(1-\eta) a^{2} B^{2} / 2} \leqq \frac{1}{3} \int_{\alpha}^{\beta} e^{a S} d x .
$$

Equations (4.10) and (4.9), together with (4.5) give us

$$
a J_{3}>\frac{1}{6} \int_{\alpha}^{\beta} e^{a S} d x>\frac{1}{12}(\beta-\alpha) e^{(1-\delta) a^{2} B^{2} / 2} .
$$

We now investigate $a J_{3}$ :

$$
a J_{3}=a \int_{a B^{2}(1-\delta)}^{a B^{2}(1+\delta)} e^{a y} W(y) d y \leqq 2 a^{2} B^{2} e^{a^{2} B^{2}(1+\delta)} W\left(a B^{2}(1-\delta)\right) .
$$

Hence, from (4.11) wehave $12^{-1}(\beta-\alpha) e^{(1-\delta) a^{2} B^{2} / 2} \leqq 2 a^{2} B^{2} e^{a^{2} B^{2}(1+\delta)} W\left(a B^{2}(1-\delta)\right)$. This implies

$$
W\left(a B^{2}(1-\delta)\right) \geqq(\beta-\alpha)\left(24 a^{2} B^{2}\right)^{-1} e^{-(1+3 \delta) a^{2} B^{2} / 2} .
$$

Since if $B$ is so large that $\log 24 a^{2} B^{2}<a^{2} B^{2} \delta / 2$,

$$
\begin{aligned}
W\left(a B^{2}(1-\delta)\right) & >(\beta-\alpha) e^{-(1+4 \delta) a^{2} B^{2} / 2} \\
& \geqq(\beta-\alpha) e^{-\left(1-\epsilon^{\prime}\right)^{2}(1+4 \delta) \log \log B}=(\beta-\alpha) / \log B .
\end{aligned}
$$

Since $a B^{2}(1-\delta)=\left(1-\epsilon^{\prime}\right)(1-\delta)\left(2 B^{2} \log \log B\right)^{1 / 2}=(1-\epsilon)\left(2 B^{2} \log \log B\right)^{1 / 2}$, equation (4.12) gives us the conclusion of Lemma 2 .

5 . We will now outline the proof of Theorem $1^{\prime}$ :

We begin by cutting up our series $S(x)=\sum_{\nu=1}^{\infty} a_{\nu} \cos n_{\nu} x$ into successive disjoint blocks of terms in a manner which we will now explain. We let

$$
\widetilde{B}_{N_{j}}=\left(\frac{1}{2} \sum a_{v}^{2}\right)^{1 / 2}
$$

where the sum is taken over all the coefficients in the $j$ th block. Let $c$ be a large number which will be chosen later. We define the first block of terms to be the first terms of the series $S(x)$ taken until $\widetilde{B}_{N_{1}} \geqq c$. We note 


$$
1 \leqq \frac{\tilde{B}_{N_{1}}^{2}}{c^{2}} \leqq \frac{\tilde{B}_{N_{1}-1}^{2}}{c^{2}}+\frac{1}{2} \frac{a_{N_{1}}^{2}}{c^{2}} \leqq 1+\frac{a_{N_{1}}^{2}}{c^{2}}
$$

and since $a_{k} / B_{k} \rightarrow 0, a_{N_{1}}^{2} / c^{2}$ is very small if only $c$ is large. Thus $1 \leqq \widetilde{B}_{N_{1}}^{2} / c^{2}$ $\leqq 1+\gamma$ where $\gamma$ can be taken as small as we like if only $c$ is large enough, and hence

$$
1 \leqq \frac{\widetilde{B}_{N_{1}}}{c} \leqq 1+\gamma
$$

The $j$ th block of terms starts where the $j-1$ st leaves off and is taken until

$$
1 \leqq \frac{\tilde{B}_{N_{j}}}{c^{j}}
$$

Thus

$$
1 \leqq \frac{\widetilde{B}_{N_{j}}}{c^{j}} \leqq 1+\gamma
$$

The $j$ th block of terms, which we denote by $F_{j}$, and hence the number $N_{j}$, is thus defined inductively.

Clearly, if $c$ is very large, then in some sense the $j$ th block of terms is the major portion of the partial sum $S_{N_{j}}$, and we would expect it to determine the behavior of $S_{N_{j}}$. We let

$$
X_{j}=\left(2 B_{N_{j}}^{2} \log \log B_{N_{j}}\right)^{1 / 2}
$$

and consider $S_{N_{j}} / X_{j}$. We wish to show that there exists a number $k$ such that

$$
\sup _{1 \leqq j \leqq k} \frac{S_{N_{j}}(x)}{X_{j}} \geqq 1-\epsilon
$$

for $x$ in a set of measure greater than $2 \pi-\delta$. Clearly,

$$
\frac{S_{N_{j}}(x)}{X_{j}}=\frac{F_{j}(x)}{X_{j}}+\frac{S_{N_{j-1}}(x)}{X_{j}} .
$$

We will show that if $c$ (and hence $\widetilde{B}_{N_{j}}$ ) is taken large enough, then there exists a number $k$ such that

$$
\sup _{1 \leqq j \leqq k} \frac{F_{j}(x)}{X_{j}} \geqq 1-\epsilon / 2
$$

in a set of measure nearly $2 \pi$, and that the term $S_{N_{j-1}} / X_{j}$ is very small in a set of measure nearly $2 \pi$. 
6. Before we can proceed in the manner just outlined we must put our series $S(x)$ into a form such that Lemma's 1 and 2 are applicable to it. We will show that a partial sum $S_{N}(x)$ of $S(x)$ can be cut into two series, $S_{N}^{\prime}$ and $S_{N}^{\prime \prime}$, each satisfying condition $(r, R)$ and that this can be done in such a way that the series $S_{N}^{\prime \prime}$ is "small" in comparison with $S_{N}^{\prime}$. This result is contained in the following lemma.

Lemma 3. Let $S(x)=\sum a_{k} \cos n_{k} x$ be a finite lacunary series and let $B^{2}$ $=2^{-1} \sum a_{k}^{2}$. Let $r=r(q)$ be defined by the following inequalities

$$
\left(q^{r / 3}-1\right)^{-1} \leqq(q-1) / 4 ; \quad q^{-r / 3}(R+1)^{2} \leqq 1 / 2 .
$$

Let $R$ have the property that

$$
\frac{r}{R} \leqq \frac{\epsilon^{2}}{96}
$$

and also the property that it is divisible by $3 r$.

Then there exist series $S^{\prime}$ and $S^{\prime \prime}$ such that $S^{\prime}+S^{\prime \prime}=S$ and the series $S^{\prime}$ consists of blocks of length contained between $R / 3$ and $R$, separated by empty blocks of length $r$. Furthermore, if we write $B^{2}=B^{\prime 2}+B^{\prime \prime 2}$, then

$$
\frac{B^{\prime \prime 2}}{B^{2}} \leqq \frac{\epsilon^{2}}{32} \text {. }
$$

We begin by subdividing $S$ into successive blocks of terms each of length $R / 3$. (If the length of $S$ is not divisible by $R / 3$ we complete $S$ by zeros.) In blocks with odd indices we select subblocks of length $r$ in such a way that, if $\beta_{n}^{2}$ is the sum of the $a_{k}^{2}$ taken over the $h$ th odd block, and $\beta_{h}^{\prime \prime 2}$ is the sum of the $a_{k}^{2}$ taken over its subblock of length $r$, then

$$
\left(\beta_{h}^{\prime \prime} / \beta_{h}\right)^{2} \leqq r /(R / 3) \text {. }
$$

(This is possible since $R / 3$ is divisible by $r$. We take for our subblock that successive group of terms which minimizes $\beta_{h}^{\prime \prime 2}$.)

We denote the blocks of length $r$ successively by $\Delta_{1}^{\prime \prime}, \Delta_{2}^{\prime \prime}, \ldots$. If we remove them from $S$, the remainder consists of blocks of length between $R / 3$ and $R$, which we denote by $\Delta_{1}^{\prime}, \Delta_{2}^{\prime}, \cdots$. Clearly, $S^{\prime}=\sum \Delta_{i}^{\prime}$ and $S^{\prime \prime}=\sum \Delta_{i}^{\prime \prime}$ satisfy the requirements of the lemma.

7. Before turning the proof of Theorem 1 as outlined in $\$ 5$, we will collect the terminology we need, restating old definitions or introducing new ones as necessary.

We assume that the series $S(x)$ of Theorem $1^{\prime}$;

$$
S(x)=\sum_{\nu=1}^{\infty} a_{\nu} \cos n_{\nu} x
$$

has been divided into blocks of terms in the manner demonstrated in $\S 5$. Then 
(i) $F_{j}(x)$ is the sum of the terms of the $j$ th block

(ii) $\widetilde{B}_{N_{j}}$ is equal to $\left(2^{-1} \sum a_{\nu}^{2}\right)^{1 / 2}$, where the sum is taken over the indices of the $j$ th block.

(iii) $S_{N_{j}}(x)$ is the sum of the terms in the first $j$ blocks of the series (7.1). We note that it is a partial sum of (7.1).

(iv) $S_{N_{j}}^{\prime}(x)$ is the subseries of $S_{N_{j}}(x)$ determined by Lemma 3 , consisting of groups of terms of length between $R / 3$ and $R$.

(v) $S_{N_{j}}^{\prime \prime}(x)$ is the subseries of $S_{N_{j}}(x)$ determined by Lemma 3 , consisting of groups of terms of length $r$.

(vi) $B_{N_{j}}^{\prime}$ is equal to $\left(2^{-1} \sum a_{\nu}^{2}\right)^{1 / 2}$, where the sum is taken over the indices of $S_{N_{j}}^{\prime}$.

(vii) $B_{N_{j}}^{\prime \prime}$ is equal to $\left(2^{-1} \sum a_{\nu}^{2}\right)^{1 / 2}$, where the sum is taken over the indices of $S_{N_{j}}^{\prime \prime}$.

(viii) $F_{j}^{\prime}$ is the sum of those terms of the series (7.1) which belong to both $F_{j}$ and $S_{N_{j}}^{\prime}$. of $F_{j}^{\prime}$.

(ix) $\widetilde{B}_{N_{j}}^{\prime}$ is equal to $\left(2^{-1} \sum a_{\nu}^{2}\right)^{1 / 2}$, where the sum is taken over the indices

(x) $X_{j}$ is equal to $\left(2 B_{N_{j}}^{2} \log \log B_{N_{j}}\right)^{1 / 2}$.

(xi) $\tilde{X}_{j}^{\prime}$ is equal to $\left(2 \widetilde{B}_{N_{j}}^{\prime 2} \log \log \widetilde{B}_{N_{j}}^{\prime}\right)^{1 / 2}$.

8. Using the definitions of the preceding section we obtain the following modified form of (5.3):

$$
\frac{S_{N_{j}}}{X_{j}}=\frac{F_{j}^{\prime}}{\tilde{X}_{j}^{\prime}} \frac{\tilde{X}_{j}^{\prime}}{X_{j}}+\frac{S_{N_{j-1}}^{\prime}}{2^{-1 / 2} X_{j-1}} \frac{2^{-1 / 2} X_{j-1}}{X_{j}}+\frac{S_{N_{j}}^{\prime \prime}}{X_{j}} .
$$

By inspection of (8.1) we can see that Theorem $1^{\prime}$ will be proved if we show that for $c$ large enough (we recall that the splitting of $S(x)$ into blocks depends upon the choice of $c$ ) there exists a number $k$ such that the following inequalities hold:

$$
\sup _{1 \leqq j \leqq k} \frac{F_{j}^{\prime}(x)}{\tilde{X}_{j}^{\prime}} \geqq(1-\epsilon / 4)
$$

for all $x$ in a set of measure greater than $2 \pi-\delta / 4$.

$$
\begin{aligned}
\frac{X_{j}^{\prime}}{X_{j}} & \geqq(1-\epsilon / 4) . \\
\frac{\left|S_{N_{j-1}}^{\prime}(x)\right|}{2^{1 / 2} X_{j-1}} & \leqq 1
\end{aligned}
$$

for all $j$ and all $x$ in a set of measure greater than $2 \pi-\delta / 4$. 


$$
\begin{array}{r}
\frac{2 X_{j-1}}{X_{j}} \leqq \epsilon / 4, \\
\frac{\left|S_{N_{j}}^{\prime \prime}(x)\right|}{X_{j}} \leqq \epsilon / 4
\end{array}
$$

for all $j$ and all $x$ in a set of measure greater than $2 \pi-\delta / 4$.

9. The demonstration of points (iii) and ( $v$ ) of the preceding section will be contained in the following two lemmas.

Lemma 4. If

$$
B_{N_{j}}^{-1}\left(2 \log \log B_{N_{j}}\right)^{1 / 2} M_{N_{j}} R^{2} A \leqq .05
$$

where $M_{N_{j}}$ is the maximum of the absolute value of the coefficients from $S_{N_{j}}$, and $A$ is the constant of Lemma 1 , then

$$
\sum_{j=2}^{\infty}\left|\Gamma_{j}^{\prime}\right| \leqq(\log c)^{-1} \sum_{j=2}^{\infty}(1 / j)^{1 \cdot 4},
$$

where $\Gamma_{j}^{\prime}=\left\{x: S_{N_{j}}^{\prime}(x) / 2^{1 / 2} X_{j} \geqq 1\right\}$.

We take $\lambda=B_{N_{j}}^{-1}\left(2 \log \log B_{N_{j}}\right)^{1 / 2}, \eta=.2$ and apply Lemma 1 . We obtain (9.3) $e^{\lambda y} W(y) \leqq \int_{0}^{2 \pi} \exp \left(\lambda S_{N_{j}}^{\prime}\right) d x \leqq \exp \left((1.2) \lambda^{2}{B_{N_{j}}^{\prime 2}}^{2}\right) / 2 \leqq \exp \left((1.2) \lambda^{2} B_{N_{j}}^{2}\right) / 2$. If $y=2^{1 / 2}\left(2 B_{N_{j}}^{2} \log \log B_{N_{j}}\right)^{1 / 2}$, equation (9.3) yields

$$
\begin{aligned}
W(y) & \leqq \exp \left((1.2) \log \log B_{N_{j}}-2^{1 / 2} \log \log B_{N_{j}}\right) \\
& \leqq \exp \left((1.4) \log \log B_{N_{j}}\right) \leqq\left(\log B_{N_{j}}\right)^{-1.4} \leqq(\log c)^{-1}(1 / j)^{1.4} .
\end{aligned}
$$

Since $W(y)=\left|\Gamma_{j}^{\prime}\right|$ for the value of $y$ we have chosen, the conclusion of our lemma follows.

Lemma 5. If

$$
\frac{4}{\epsilon} \frac{\left(2 \log \log B_{N_{j}}\right)^{2}}{B_{N_{j}}} M_{N_{j}} R^{2} A \leqq .05
$$

then

$$
\sum_{j=1}\left|\Gamma_{j}^{\prime \prime}\right| \leqq(\log c)^{-1} \sum_{j=1}(1 / j)^{1.4}
$$

where

$$
\Gamma_{j}^{\prime \prime}=\left\{x: S_{N_{j}}^{\prime \prime}(x) / X_{j} \geqq \epsilon / 4\right\}
$$


We take

$$
\lambda=\frac{4}{\epsilon} \frac{\left(2 \log \log B_{N_{j}}\right)^{1 / 2}}{B_{N_{j}}} ; \quad \eta=.2
$$

and apply Lemma 1 . We have

$$
e^{\lambda} y W(y) \leqq \int_{0}^{2 \pi} \exp \left(\lambda S_{N_{j}}^{\prime \prime}\right) d x \leqq \exp \left((1.2) \lambda^{2}{B_{N_{j}}^{\prime \prime}}^{2}\right) / 2 .
$$

Hence

$$
\begin{aligned}
W(y) \leqq & \exp (1.2)(4 / \epsilon)^{2}\left(\log \log B_{N_{j}}\right)\left(B_{N_{j}}^{\prime \prime} / B_{N_{j}}\right)^{2} \\
& -(4 / \epsilon)\left(2 \log \log B_{N_{j}}\right)^{1 / 2} y / B_{N_{j}} .
\end{aligned}
$$

If $y=(\epsilon / 4)\left(2 B_{N_{j}}^{2} \log \log B_{N_{j}}\right)^{1 / 2}$, equations (9.7) and (6.3) yield

$$
\begin{aligned}
W(y) & \leqq \exp \left(.6 \log \log B_{N_{j}}-2 \log \log B_{N_{j}}\right) \leqq \exp \left(-(1.4) \log \log B_{N_{j}}\right) \\
& \leqq\left(\log B_{N_{j}}\right)^{-1.4} \leqq(\log c)^{-1}(1 / j)^{1.4},
\end{aligned}
$$

and since $W(y)=\left|\Gamma_{j}^{\prime \prime}\right|$, our lemma has been proved.

10. In this section we will consider points (ii) and (iv) of $\S 8$. Clearly,

$$
\begin{aligned}
\frac{2^{1 / 2} X_{j-1}}{X_{j}} & =2^{1 / 2}\left(\frac{B_{N_{j-1}}^{2} \log \log B_{N_{j-1}}}{B_{N_{j}}^{2} \log \log B_{N_{j}}}\right)^{1 / 2} \leqq 2^{1 / 2} \frac{B_{N_{j-1}}}{B_{N_{j}}} \\
& \leqq 2(1+\gamma)\left(\frac{\sum_{i=1}^{j-1} c^{2 i}}{\sum_{i=1}^{j} c^{2 i}}\right)=\frac{2^{1 / 2}}{c}(1+\gamma)\left(\frac{c^{2(j-1)}-1}{c^{2(j-1)}-1 / c^{2}}\right)^{1 / 2} \\
& =(1+\gamma) \frac{2^{1 / 2}}{c} .
\end{aligned}
$$

Thus, we have

$$
\frac{2^{1 / 2} X_{j-1}}{X_{j}} \leqq(1+\gamma) \frac{2^{1 / 2}}{c}
$$

We now investigate

$$
\frac{\widetilde{X}_{j}^{\prime}}{X_{j}}=\left(\frac{\widetilde{B}_{N_{j}}^{\prime 2} \log \log \widetilde{B}_{N_{j}}}{\sum_{i=1}^{j} B_{N_{j}}^{2} \log \log \left(\sum_{i=1}^{j} B_{N_{i}}^{2}\right)^{1 / 2}}\right)^{1 / 2} .
$$

We have 
$(10.2)$

$$
\left(\frac{{\bar{B}_{N j}^{\prime}}^{\prime 2}}{\sum_{i=1}^{j} B_{N_{i}}^{2}}\right)^{1 / 2} \geqq\left(\frac{\left(1-\epsilon^{2} / 32\right) B_{N_{j}}^{2}}{\sum_{i=1}^{j} B_{N_{i}}^{2}}\right)^{1 / 2} \geqq\left(\frac{\left(1-\epsilon^{2} / 32\right) c^{2 j}}{(1+\gamma) \sum_{i=1}^{j} c^{2 i}}\right)^{1 / 2}
$$

$$
\geqq\left(\frac{1-\epsilon^{2} / 32}{1+\gamma}\right)^{1 / 2}\left(\frac{c^{2}-1}{c^{2}}\right)^{1 / 2}=g(\epsilon, \gamma, c) .
$$

We note that $g(\epsilon, \gamma, c)$ tends to 1 as $\epsilon, \gamma, 1 / c$ tend to zero. Clearly,

$$
\frac{\tilde{X}_{j}^{\prime}}{X_{j}} \geqq g\left(\frac{\log \log \left(\sum_{i=1}^{j} B_{N_{i}}^{2}\right)^{1 / 2} g}{\log \log \left(\sum_{i=1}^{j} B_{N_{i}}^{2}\right)}\right)^{1 / 2} \geqq 1-\epsilon / 4
$$

provided $g(\epsilon, \gamma, c)$ is close enough to 1 .

11. This section, which deals with (i) of $\S 8$, is a somewhat modified form of a portion of the paper of Erdös and Gál [1]. We define

$$
\begin{aligned}
& E_{1}=\left\{x ; x \in(0,2 \pi), F_{1}^{\prime}(x) \geqq(1-\epsilon / 4) \tilde{X}_{j}^{\prime}\right\}, \\
& E_{j}=\left\{x ; x \in(0,2 \pi)-\left(E_{1}+E_{2}+\cdots+E_{j-1}\right) ;\right. \\
& \left.\quad F_{j}^{\prime}(x) \geqq(1-\epsilon / 4) \tilde{X}_{j}^{\prime}\right\} .
\end{aligned}
$$

From now on we will denote $(0,2 \pi)$ by $I$.

Since $F_{j}^{\prime}$ is a polynomial of degree $N_{j}$, the set $\left\{x ; x \in I ; F_{j}(x) \leqq(1-\epsilon / 4) \tilde{X}_{j}^{\prime}\right\}$ consists of at most $2 N_{j}$ intervals. In particular $I-E_{1}$ consists of at most $\rho_{1}$ intervals where $\rho_{1} \leqq 2 N_{1}$. It is easily seen that the set $I-\left(E_{1}+E_{2}+\cdots+E_{j}\right)$ consists of $\rho_{j}$ intervals where $\rho_{j} \leqq 2\left(N_{1}+N_{2}+\cdots+N_{j}\right) \leqq A N_{j}$, where $A=2 \sum_{n=0}^{\infty}(1 / q)^{n}$. If we let $e_{j+1}(j \geqq 1)$ be the union of those intervals of $I-\left(E_{1}+E_{2}+\cdots+E_{j}\right)$ the length of which is less than $\theta / A N_{j}$ where

$$
\theta=\left(\frac{\delta}{32 \pi}\right)\left(1-\log \frac{\delta}{8 \pi}\right)^{-1}
$$

we see that if $\lambda M R^{2} A<\theta / 2$, the estimate of Lemma 2 can be applied to each of the intervals of $I-\left(E_{1}+\cdots+E_{j}\right)-e_{j+1}$ and hence to $I-\left(E_{1}+\cdots+E_{j}\right)$ $-e_{j+1}$. By Lemma 2,

$$
\begin{aligned}
&\left|E_{j+1}\right| \geqq \frac{\left|I-\left(E_{1}+E_{2}+\cdots+E_{j}\right)-e_{j+1}\right|}{\log \widetilde{B}_{N_{j+1}}^{\prime}} \\
&\left|E_{1}\right| \geqq \frac{2 \pi}{\log \widetilde{B}_{N_{1}}^{\prime}} .
\end{aligned}
$$

From the above inequalities we obtain by induction on $k$ 


$$
\left|I-\left(E_{1}+\cdots+E_{k}\right)\right| \leqq 2 \pi I_{j=1}^{k}\left(1-\frac{1}{\log \widetilde{B}_{N_{j}}^{\prime}}\right)+2 \pi \sum_{j=1}^{k} \frac{\left|e_{j+1}\right|}{\log \widetilde{B}_{N_{j}}^{\prime}} .
$$

Since $\log \widetilde{B}_{N_{j}}^{\prime} \geqq(1 / 2) \log B_{N_{j}}$ the inequality (11.6) gives us

$$
\begin{aligned}
\left|I-\left(E_{1}+\cdots+E_{k}\right)\right| & \leqq 2 \pi \prod_{j=1}^{k}\left(1-\frac{1}{\log B_{N_{j}}}\right)+2 \pi \sum_{j=1}^{k} \frac{\left|e_{j+1}\right|}{2^{-1} \log B_{N_{j}}} \\
& \leqq 2 \pi \prod_{j=1}^{k}\left(1-\frac{1}{\log (1+\gamma) c^{j}}\right)+2 \pi \sum_{j=1}^{k} \frac{2\left|e_{j+1}\right|}{\log c^{j}} \\
& \leqq 2 \pi \prod_{j=1}^{k}\left(1-\frac{1}{2 j \log c}\right)+2 \pi \sum_{j=1}^{k} \frac{2\left|e_{j+1}\right|}{j \log c} .
\end{aligned}
$$

Now we choose a positive number $p$ such that

$$
2 \pi e^{-p} \leqq \delta / 4 \text {. }
$$

Then we choose $k$ in such a way that

$$
p+1 \geqq \sum_{j=1}^{k} \frac{1}{2 j \log c} \geqq p
$$

Thus

$$
2 \pi \prod_{j=1}^{k}\left(1-\frac{1}{2 j \log c}\right) \leqq 2 \pi\left(\exp \left(-\sum_{j=1}^{l} \frac{1}{2 j \log c}\right)\right) \leqq 2 \pi e^{-p} \leqq \delta / 4
$$

Since $\left|e_{j+1}\right| \leqq A N_{j} \theta / A N_{j}=\theta$, from (11.3) we have

$$
\begin{aligned}
2 \pi \sum_{j=1}^{k} \frac{2\left|e_{j+1}\right|}{j \log c} & \leqq 4 \pi \theta \sum_{j=1}^{k} \frac{1}{j \log c} \leqq 8 \pi \theta(p+1) \\
& \leqq 8 \pi \theta\left(1-\log \frac{\delta}{8 \pi}\right) \leqq \delta / 4
\end{aligned}
$$

From (11.7), (11.10), and (11.11) we obtain

$$
\left|I-\left(E_{1}+\cdots+E_{k}\right)\right| \leqq \delta / 2 .
$$

12. Now we will put all the preceding results together to obtain a proof of Theorem $1^{\prime}$ according to the plan of $\S 8$. We recall that

$$
\frac{S_{N_{j}}}{X_{j}} \geqq \frac{F_{j}^{\prime}}{\tilde{X}_{j}} \frac{\tilde{X}_{j}^{\prime}}{X_{j}}-\frac{S_{N_{j-1}}}{2^{1 / 2} X_{j-1}} \frac{2^{1 / 2} X_{j-1}}{X_{j}}-\frac{\left|S_{N_{j}}^{\prime \prime}\right|}{X_{j}} .
$$

We first choose $c$ and hence $B_{N_{j}}$, so large that 


$$
\begin{gathered}
(4 / \epsilon) B_{N_{j}}^{-1}\left(2 \log \log B_{N_{j}}\right) M_{N_{j}} R^{2} A \leqq .05 ; \\
(2 / \log c) \sum(1 / j)^{1.4} \leqq \delta / 4 ; \quad(1+\gamma) 2^{1 / 2} / c \leqq \epsilon / 4, \\
g \frac{\log \log g c}{\log \log c} \geqq 1-\epsilon / 4,
\end{gathered}
$$

where $g$ has the meaning of $\S 10$, and

$$
20 B_{N_{j}}^{-1}\left(2 \log \log B_{N_{j}}\right)^{1 / 2} M_{N_{j}} R^{2} A \leqq \theta / 4 .
$$

Now (12.2) ensures the applicability of Lemma's 4 and 5, from which we obviously obtain

$$
\frac{\left|S_{N_{j-1}}^{\prime}\right|}{2^{1 / 2} X_{j-1}} \leqq 1
$$

on a set of measure $\geqq 2 \pi-\delta / 4$, and

$$
\left|S_{N_{j}}^{\prime \prime}\right| X_{j}^{-1} \leqq \epsilon / 4
$$

on a set of measure $\geqq 2 \pi-\delta / 4$.

From (10.1) and (10.3) we see that (12.4) and (12.5) above insure us that

$$
\frac{\tilde{X}_{j}^{\prime}}{X_{j}} \geqq(1-\epsilon / 4) ; \quad \frac{2^{1 / 2} X_{j-1}}{X_{j}} \leqq \epsilon / 4 .
$$

Equations (12.4), (12.5), and (12.6) are simply points (ii), (iii), (iv) and (v) of $\S 8$.

After we have fixed $c$ large enough to satisfy all the above inequalities and also to ensure that $N_{1}$ is as large as we desire, we then pick $k$ to satisfy the inequality

$$
(p+1) \geqq \sum_{j=1}^{k} \frac{1}{2 j \log c} \geqq p
$$

and hence, by $\$ 11$, we have

$$
\sup _{1 \leqq j \leqq k} \frac{F_{j}^{\prime}}{\tilde{X}_{j}^{\prime}} \geqq 1-\epsilon / 4
$$

on a set of measure $\geqq 2 \pi-\delta / 4$. Since this latter inequality is (i) of $\S 8$, we have demonstrated all points of $\S 8$, and thus we have proved Theorem $1^{\prime}$.

13. We now pass to the proof of the inequality

$$
\lim \sup \frac{S_{N}(x)}{\left(2 B_{N}^{2} \log \log B_{N}\right)^{1 / 2}} \leqq 1 \text { a.e. }
$$


As was stated in $\S 1$, the result (13.1) has already been established by Salem and Zygmund in the case of $n_{k}$ integral. If the $n_{k}$ are not all integral their proof requires modification since the system $\left\{e^{i n_{k} x}\right\}$ is then no longer orthogonal over the interval $(-\pi, \pi)$.

Our modification is based on the well known fact that if $\left|n_{k}-n_{l}\right| \geqq 2 h>0$ for all $k \neq 1$, then the functions

$$
e^{i n_{k} x} \frac{\sin h x}{x}
$$

are orthogonal over the interval $(-\infty,+\infty)$ or what is the same thing, the system $\left\{e^{i n k x}\right\}$ is orthogonal over $(-\infty,+\infty)$ with respect to the weight function $x^{-2} \sin ^{2} h x$.

If we use this fact some of the arguments of Salem and Zygmund become immediately evident. Others such as those used in Lemma 6 below need no modification at all. However, to make this presentation self-contained we give all the proofs.

14. Leмma 6. Let $\theta$ be any fixed number greater than 1 . Let $N_{j}$ be the first number such that

$$
\theta^{j} \leqq 2 B_{N_{j}}^{2} \leqq \theta^{j+1}
$$$$
i=1,2, \cdots
$$

The number exists for $j$ large enough and we have a.e. in $x$,

$$
\limsup _{j \rightarrow \infty} \frac{S_{N_{j}}(x)}{\left(2 B_{N_{j}}^{2} \log \log B_{N_{j}}\right)^{1 / 2}} \leqq 1 .
$$

If $N_{j}$ does not exist for $j$ large enough then there exist arbitrarily large values of $j$ such that the interval $\left(\theta^{j}, \theta^{j+1}\right)$ contains no number $2 B_{m}^{2}$ but such that the interval $\left(\theta^{i+1}, \theta^{i+2}\right)$ contains one such number. Thus

$$
2 B_{m-1}^{2} \leqq \theta^{j} ; \quad \theta^{j+2}>2 B_{m}^{2} \geqq \theta^{j+1}
$$

which imply

$$
a_{m}^{2} \geqq \theta^{j+1}-\theta^{j}=\theta^{j}(\theta-1)
$$

and hence

$$
\frac{a_{m}^{2}}{2 B_{m}^{2}} \geqq \frac{\theta^{j}(\theta-1)}{\theta^{i+2}}=\frac{\theta-1}{\theta^{2}} .
$$

This last inequality contradicts the hypotheses $M_{N}=o\left(B_{N}\right)$. This proves the first part of Lemma 6.

Next we fix an $\epsilon>0$ and split $S_{N_{j}}$ into two series, $S_{N_{j}}^{\prime}$ and $S_{N_{j}}^{\prime \prime}$, with the 
properties mentioned in the statement of Lemma 3 . We let

$$
\lambda=\frac{\left(2 \log \log B_{N_{j}}\right)^{1 / 2}}{B_{N_{j}}}, \quad \eta=\epsilon
$$

and consider only $j$ so large that the hypotheses of Lemma 1 are satisfied by $S_{N_{i}}^{\prime}$. Then, according to Lemma 1 ,

$$
\begin{aligned}
\int_{\alpha} \exp \left(\lambda S_{N_{j}}^{\prime \prime}\right) d x & \leqq \frac{3}{2}(\beta-\alpha)\left(\exp \left((1+\epsilon) \lambda^{2}{B_{N_{j}}^{\prime}}^{2} / 2\right)\right) \\
& \leqq \frac{3}{2}(\beta-\alpha)\left(\exp \left((1+\epsilon) \lambda^{2} B_{N_{j}}^{2}\right)\right) / 2 .
\end{aligned}
$$

Now if $\Gamma_{j}=\left\{x: S_{N_{j}}^{\prime}(x) \geqq(1+\epsilon)\left(2 B_{N_{j}}^{2} \log \log B_{N_{j}}\right)^{1 / 2}\right\}$, then

$$
\left|\Gamma_{j}\right| \exp \left\{(1+\epsilon)\left(2 B_{N_{j}}^{2} \log \log B_{N_{j}}\right)^{1 / 2} \lambda\right\} \leqq \frac{3}{2}(\beta-\alpha)\left(\exp \left((1+\epsilon) \lambda^{2} B_{N_{j}}^{2}\right)\right) / 2 .
$$

Substituting for $\lambda$, its value given above, we see that

$$
\left|\Gamma_{j}\right| \leqq \frac{3}{2}(\beta-\alpha) e^{-(1+\epsilon) \log \log B_{N j}}
$$

from which, and the inequality $2 B_{N_{j}} \geqq \theta^{j}$ it follows that $\sum_{j=1}^{\infty}\left|\Gamma_{j}\right|<\infty$. This last inequality implies, a.e. in $x$,

$$
\lim \sup \frac{S_{N_{j}}^{\prime}(x)}{\left(2 B_{N_{j}}^{2} \log \log B_{N_{j}}\right)^{1 / 2}} \leqq 1+\epsilon .
$$

Now by an argument which follows very closely that of Lemma 5, we can show, a.e. in $x$,

$$
\lim \sup \frac{S_{N_{j}}^{\prime \prime}(x)}{\left(2 B_{N_{j}}^{2} \log \log B_{N_{j}}\right)^{1 / 2}} \leqq \epsilon .
$$

Hence

$$
\lim \sup \frac{S_{N_{j}}(x)}{\left(2 B_{N_{j}}^{2} \log \log B_{N_{j}}\right)^{1 / 2}} \leqq 1+2 \epsilon .
$$

Since $\epsilon$ is arbitrary, we have the conclusion of the lemma.

15. Lemma 7. Let

$$
S(x)=\sum_{k=1}^{N} a_{k} \cos n_{k} x ; \quad \frac{n_{k+1}}{n_{k}}>q>1
$$


be a lacunary trigonometric polynomial satisfying $\left|n_{k}-n_{l}\right| \geqq 2 k \neq l$ and let

$$
S^{*}(x)=\max _{K \leqq N}\left|S_{K}(x)\right| .
$$

Then if $(\alpha, \beta)$ is any interval of length less than 1

$$
\int_{\alpha}^{\beta} \exp \left(S^{*}(x)\left|\frac{\sin x}{x}\right|\right) d x \leqq 32 \int_{-\infty}^{\infty}\left(\frac{\sin x}{x}\right)^{2} e^{A q|S(x)|} d x
$$

where $A_{q}$ is a constant depending only on $q$.

Now if

$$
\begin{gathered}
f(x)=\frac{\sin x}{x} \sum_{k=1}^{N} a_{k} \cos n_{k} x, \\
g(x)=\left\{\begin{array}{l}
a_{k}: x \in\left(n_{k}-1, n_{k}+1\right) \text { or }\left(-n_{k}-1,-n_{k}+1\right), \\
0: \text { otherwise; }
\end{array}\right.
\end{gathered}
$$

then clearly

$$
f(x)=\frac{1}{4} \int_{-\infty}^{\infty} e^{-i \lambda x} g(\lambda) d x
$$

and

$$
f_{w}(x)=\frac{\sin x}{x} \sum_{n_{k} \leq w} a_{k} \cos n_{k} x=\frac{1}{4} \int_{-w}^{w} e^{-i \lambda x} g(\lambda) d x=\frac{\sin x}{x} S_{w}(x),
$$

where

$$
S_{w}(x)=\sum_{n_{k} \leq w} a_{k} \cos n_{k} x
$$

Furthermore

$$
\begin{aligned}
\frac{\sin x}{x}-\frac{1}{w} \int_{-w}^{w} S_{\delta}(x) d \delta & =\frac{1}{2} \int_{-w}^{w} e^{-i \lambda x} g(\lambda)(1-|\lambda| / w) d \lambda \\
& =C \int_{-\infty}^{\infty} f(x-\lambda) \frac{\sin ^{2} w \lambda / 2}{w \lambda^{2}} d \lambda,
\end{aligned}
$$

where $C$ is a constant.

Applying to the last term of the above equality an argument which follows very closely that on page 248 of Zygmund [1] we see that

$$
\max _{w} c\left|\int_{-\infty}^{\infty} f(x-\lambda) \frac{\sin ^{2}(w \lambda / 2)}{w \lambda^{2}} d \lambda\right| \leqq A M(f ; x)
$$


where $M(x ; f)$ if the well known Hardy-Littlewood maximal function defined by

$$
M(f ; x)=\max \frac{1}{\xi-x} \int_{x}^{\xi}|f(t)| d t
$$

Thus

$$
\left|\frac{\sin x}{x}\right| \max _{w}\left|\frac{1}{w} \int_{-w}^{w} S_{\delta}(x) d \delta\right| \leqq A M(f ; x) .
$$

Since

we have

$$
\begin{aligned}
\int_{-n_{k}}^{n_{k}} S_{\delta}(x) d \delta & =\int_{-n_{k-1}}^{n_{k-1}} S_{\delta}(x) d \delta+2 \int_{n_{k-1}}^{n_{k}} S_{\delta}(x) d \delta \\
& =\int_{-n_{k-1}}^{n_{k-1}} S_{\delta}(x) d \delta+2\left(n_{k}-n_{k-1}\right) S_{n_{k-1}}(x)
\end{aligned}
$$

$$
\begin{aligned}
S_{n_{k-1}}(x)= & \frac{n_{k}}{2\left(n_{k}-n_{k-1}\right)} \frac{1}{n_{k}} \int_{-n_{k}}^{n_{k}} S_{\delta}(x) d \delta \\
& -\frac{n_{k-1}}{\left(2 n_{k}-n_{k-1}\right)} \frac{1}{n_{k-1}} \int_{-n_{k-1}}^{n_{k-1}} S_{\delta}(x) d \delta .
\end{aligned}
$$

Now since $\left|n_{k} /\left(n_{k}-n_{k-1}\right)\right| \leqq A_{q}^{\prime}$, equation (15.3) becomes

$$
\max _{n_{k}}\left|S_{n_{k-1}}(x)\right| \leqq A_{q}^{\prime} \max _{n_{k}}\left|\frac{1}{n_{k}} \int_{-n_{k}}^{n_{k}} S_{\delta}(x) d \delta\right|,
$$

and hence

$$
\left|\frac{\sin x}{x}\right| \max \left|S_{n_{k}}(x)\right| \leqq A_{q}^{\prime} \Lambda M(f ; x)=A_{q} M(f ; x),
$$

or

$$
\left|\frac{\sin x}{x}\right| S^{*}(x) \leqq A_{q} M(f ; x) .
$$

We recall the well known fact that if $f \in L^{p}, p>1$, so is $M(f ; x)$ and furthermore $\int_{-\infty}^{\infty} M^{p}(f ; x) d x \leqq 2(p /(p-1))^{p} \int_{-\infty}^{\infty}|f|^{p} d x$. Using this fact and (15.4) we obtain

$$
\int_{-\infty}^{\infty}\left|\frac{\sin x}{x}\right|^{p}\left|S^{*}(x)\right|^{p} d x \leqq 2\left(\frac{p}{p-1}\right)^{p} \int_{-\infty}^{\infty} A_{q}^{p}\left|\frac{\sin x}{x}\right|^{p}|S(x)|^{p} d x .
$$


Thus for $p \geqq 2$,

$$
\int_{-\infty}^{\infty}\left|\frac{\sin x}{x}\right|^{p}\left|S^{*}(x)\right|{ }^{p} d x \leqq 8 \int_{-\infty}^{\infty} A_{q}^{p}\left|\frac{\sin x}{x}\right|^{p}|S(x)|^{p} d x .
$$

From this last basic inequality it follows that

$$
\begin{aligned}
\int_{\alpha}^{\beta} \exp \left(\left|\frac{\sin x}{x}\right| S^{*}\right) d x & \leqq 2 \int_{\alpha}^{\beta} \cosh \left(\left|\frac{\sin x}{x}\right| S^{*}(x)\right) d x \\
& \leqq 32 \int_{-\infty}^{\infty}\left|\frac{\sin x}{x}\right|^{2} e^{A} S^{S(x)} d x
\end{aligned}
$$

which is the conclusion of our lemma.

16. Lemma 8. Let

$$
S(x)=\sum_{k=1}^{N} a_{k} \cos n_{k} x
$$

be a trigonometric polynomial satisfying condition $(r, R)$ (see $\$ 3$ for a definition) where $r$ satisfies (3.1). Suppose, in addition that $n_{1}$ is greater than a certain number $n_{0}$ which depends on $q$. Let

Then

$$
M=\max _{k \leqq N}\left|a_{k}\right| .
$$

$$
\int_{-\infty}^{\infty}\left(\frac{\sin x}{x}\right)^{2} e^{A} q^{\lambda|S|} d x \leqq e^{A A_{q}^{3} M R^{2} B^{2}+A_{q}^{2} \lambda^{2} B^{2} / 2} .
$$

Let $\Delta_{h}$ and $\delta_{h}$ have the same meaning as in Lemma 1 of $\S 3$ and let

$$
w_{h}=\left(1+\lambda A_{q} \Delta_{h}+\frac{\lambda^{2} A_{q}^{2} \Delta_{h}^{2}}{2}\right) .
$$

Following exactly the proof of Lemma 1 we obtain

$$
\int_{-\infty}^{\infty}\left(\frac{\sin x}{x}\right)^{2} e^{A} \lambda^{\lambda S(x)} d x \leqq \exp \left(A A_{q}^{3} \lambda^{3} R^{2} M \sum \delta_{h}\right) \int_{-\infty}^{\infty}\left(\frac{\sin x}{x}\right)^{2} \prod w_{h} d x .
$$

Let us consider a term in the expansion of $\prod w_{h}$ whose highest nonconstant factor comes from $w_{p}$. Then just as in the proof of Lemma 1, we see that the order of such a term is greater than a constant times $\tilde{n}_{p}$. Thus if $n_{1}$ is sufficiently large our term has order greater than 2. But, for $u>2$,

$$
\int_{-\infty}^{\infty}\left(\frac{\sin x}{x}\right)^{2} \cos u x d x=0 .
$$

Thus 


$$
\int_{-\infty}^{\infty}\left(\frac{\sin x}{x}\right)^{2} \Pi w_{h} d x=\Pi\left(1+\frac{1}{2} A_{q}^{2} \lambda^{2} \delta_{h}^{2}\right) \leqq e^{A_{q}^{2} \lambda^{2} B^{2} / 2},
$$

and our lemma is demonstrated.

17. Lemma 9. Let

$$
P_{j}(x)=S_{N_{j}}(x)-S_{N_{j-2}}(x)
$$

where $S_{N_{j}}(x)$ has the meaning of Lemma 6 , and let $P_{j}^{*}(x)$ be the maximum of the absolute value of the partial sums of the polynomial $P_{j}(x)$. Then, if

$$
\beta_{j}^{2}=B_{N_{j}}^{2}-B_{N_{j-2}}^{2}
$$

and $(\alpha, \beta)$ is any interval whose closure does not contain a zero of $\sin x$, there exists a constant $c=c(q, \alpha, \beta)$ such that for almost all $x$ in $(\alpha, \beta)$,

$$
\limsup _{j \rightarrow \infty} \frac{P_{j}^{*}(x)}{\left(2 \beta_{j}^{2} \log \log \beta_{j}\right)^{1 / 2}} \leqq 2 c .
$$

We take an $r$ which satisfies (3.1) and fix an integer $R>r$. We divide $P_{j}(x)$ into successive blocks of length $R$ and take $P_{j}^{\prime}(x)$ to be the polynomial composed of the odd numbered blocks and $P_{j}^{\prime \prime}(x)$ to be the polynomial composed of the even numbered blocks.

By Lemma 6 and 7,

$$
\int_{\alpha}^{\beta} \exp \left(\lambda P_{j}^{*}(x)\left|\frac{\sin x}{x}\right|\right) d x \leqq 32 \exp \left(A A_{q}^{3} \lambda^{3} M R^{2} \beta_{j}^{2}+A_{q}^{2} \lambda^{2} \beta_{j}^{2} / 2\right) .
$$

If we take

$$
\lambda=\beta_{j}^{-1}\left(\log \log \beta_{j}\right)^{1 / 2}
$$

we see that

$$
\theta^{j} \leqq 2 B_{N_{j}}^{2} \leqq \theta^{j+1} ; \quad \theta^{j-2} \leqq 2 B_{N_{j-2}}^{2}<\theta^{j-1} ; \quad \theta^{j}-\theta^{j-1}<2 \beta_{j}^{2}<\theta^{j-1}-\theta^{j-2}
$$

and hence $\lambda=O\left(B_{N j}^{-1}\left(\log \log B_{N_{j}}\right)^{1 / 2}\right)$. But this implies $\lambda M=o(1)$, and so if $j$ is large enough we have $A A_{q} \lambda M R^{2} \leqq 1 / 2$, and hence

$$
\int_{\alpha}^{\beta} \exp \left(\lambda P_{j}^{\prime *}(x)\left|\frac{\sin x}{x}\right|\right) d x \leqq 32 \exp A_{q}^{2} \lambda^{2} \beta_{j}^{2} .
$$

Let $\gamma$ be a number such that for $x$ in $(\alpha, \beta)$

Inequality (17.3) becomes

$$
\left|\frac{\sin x}{x}\right|>\gamma>0 \text {. }
$$




$$
\int_{\alpha}^{\beta} e^{\gamma \lambda P_{j}^{\prime}(x)} d x \leqq 32 e^{A_{q}^{2} \lambda^{2} \beta_{i}^{2}}
$$

Let $\Gamma_{j}^{\prime}=\left\{x: P_{j}^{\prime *}(x)>c\left(2 \beta_{j}^{2} \log \log \beta_{j}\right)^{1 / 2}\right\}$. Then

$$
\left|\Gamma_{j}^{\prime}\right| \exp \left(\gamma \lambda c\left(2 \beta_{j}^{2} \log \log \beta_{j}\right)^{1 / 2}\right) \leqq 32 \exp \left(A_{q}^{2} \lambda^{2} \beta_{j}^{2}\right) .
$$

Upon substitution for $\lambda$ of its value given in (17.2), (17.5) becomes $\left|\Gamma_{j}^{\prime}\right|$ $\leqq 32 \exp \left(\left(A_{q}^{2}-2^{1 / 2} \gamma c\right) \log \log \beta_{j}\right)$, from which we see that if

$$
c \geqq\left(\gamma 2^{1 / 2}\right)^{-1}\left(A_{q}^{2}+2\right)
$$

then

$$
\left|\Gamma_{j}^{\prime}\right| \leqq 32 \exp \left(-2 \log \log \beta_{j}\right) \leqq 32\left(\log \beta_{j}\right)^{-2} .
$$

Since $2 \beta_{j}^{2} \geqq \theta^{j-1}(\theta-1)$, (17.6) implies $\sum_{j=1}^{\infty}\left|\Gamma_{j}^{\prime}\right|<\infty$ and hence

$$
\lim \sup \frac{P_{j}^{\prime *}(x)}{\left(2 \beta_{j}^{2} \log \log \beta_{j}\right)^{1 / 2}} \leqq C(q, \alpha, \beta) .
$$

Since

$$
P_{j}^{*}(x) \leqq P_{j}^{\prime *}(x)+P_{j}^{\prime *}(x)
$$

our lemma is established.

18. The proof of (13.1) is now almost immediate. We first note that it is sufficient to prove (13.1) for any interval $(\alpha, \beta)$ such that $\sin x$ is not zero in the closure of that interval. If $N_{j-2} \leqq m<N_{j}$, then clearly

$$
\begin{aligned}
\frac{S_{m}(x)}{\left(2 B_{m}^{2} \log \log B_{m}\right)^{1 / 2}} & \leqq \frac{\left|S_{N_{j-2}}(x)\right|}{\left(2 B_{m}^{2} \log \log B_{m}\right)^{1 / 2}}+\frac{P_{j}^{*}(x)}{\left(2 B_{m}^{2} \log \log B_{m}\right)^{1 / 2}} \\
& \leqq \frac{\left|S_{N_{j-2}}(x)\right|}{\left(2 B_{N j-2}^{2} \log \log B_{N_{j-2}}\right)^{1 / 2}}+\frac{P_{j}^{*}(x)}{\left(2 B_{N-j}^{2} \log \log B_{N_{j-2}}\right)^{1 / 2}}
\end{aligned}
$$

By Lemma 6

$$
\lim \sup \frac{\left|S_{N_{j-2}}(x)\right|}{\left(2 B_{N_{j}}^{2} \log \log B_{N_{j-2}}\right)^{1 / 2}} \leqq 1
$$

a.e. in $x$. Since

$$
2 B_{N_{j-2}}^{2} \geqq \theta^{j-2}>\frac{2 \beta_{j}^{2}}{\theta^{3}-1} ; \quad \frac{\log \log B_{N_{j-2}}}{\log \log \beta_{j}} \rightarrow 1
$$

we have, by Lemma 9 , 


$$
\begin{aligned}
\lim \sup \frac{P_{j}^{*}(x)}{\left(2 B_{N_{j-2}}^{2} \log \log B_{N_{j-2}}\right)^{1 / 2}} & \leqq\left(\theta^{3}-1\right)^{1 / 2} \lim \sup \frac{P_{j}^{*}(x)}{\left(2 \beta_{j}^{2} \log \log \beta_{j}\right)^{1 / 2}} \\
& \leqq 2 c\left(\theta^{3}-1\right)^{1 / 2} .
\end{aligned}
$$

Since $\theta$ can be chosen as close to 1 as we desire, we have

$$
\lim \sup \frac{S_{m}(x)}{\left(2 B_{m}^{2} \log \log B_{m}\right)^{1 / 2}} \leqq 1
$$

a.e. in $x$.

19. The following is an analogue of Theorem 1 for Abel means.

Theorem. Let

$$
S(r, x)=\sum_{1}^{\infty}\left(a_{k} \cos n_{k} x+b_{k} \sin n_{k} x\right) r^{n_{k}}
$$

be the Abel means of a lacunary trigonometric series. Let $B_{r}^{2}=2^{-1} \sum_{1}^{\infty}\left(a_{k}^{2}+b_{k}^{2}\right) r^{2 n_{k}}$. Then if the coefficients $a_{k}, b_{k}$ satisfy the conditions of Theorem 1, we have

$$
\limsup _{r \rightarrow 1} \frac{S(r, x)}{\left(2 B_{r}^{2} \log \log B_{r}\right)^{1 / 2}}=1
$$

almost everywhere.

This theorem is almost an immediate consequence of Theorem 1. As in Theorem 1 it is sufficient to consider cosine series. We define $N=N_{r}$ to be that integer such that

$$
r^{n_{N}} \geqq 1 / 2 ; \quad r^{n_{N}+1}<1 / 2 .
$$

We first show that $B_{r}^{2} / B_{N}^{2} \rightarrow 1$ as $r \rightarrow 1$. We write

$$
\frac{B_{r}^{2}}{B_{N}^{2}}=\frac{\frac{1}{2} \sum_{1}^{N} a_{k}^{2}}{B_{N}^{2}}+\frac{\frac{1}{2} \sum_{1}^{N} a_{k}^{2}\left(r^{2 n_{k}}-1\right)}{B_{N}^{2}}+\frac{\frac{1}{2} \sum_{m=1} a_{N+m} r^{2 n_{N+m}}}{B_{N}^{2}}=1+P+Q
$$

Since for $k \leqq n, a_{k}^{2} / B_{N}^{2} \rightarrow 0$, it follows that

$$
\begin{aligned}
& |P| \leqq \epsilon_{N} \sum_{1}^{N}\left|\left(r^{2 n_{k}}-1\right)\right| \leqq \epsilon_{N} C \sum_{1}^{N}\left(\frac{1}{q}\right)^{N-k} \rightarrow 0, \\
& |Q| \leqq 2^{-1} \sum_{m=1}^{\infty}\left(\frac{a_{N+m}}{B_{N+m}}\right)^{2}\left(\frac{B_{N+m}}{B_{N}}\right)^{2} r^{2 n} N+m .
\end{aligned}
$$

Now $B_{N+1}^{2}=a_{N+1} / 2+B_{N}^{2} \leqq 2 B_{N}^{2}$ and hence $\left(B_{N+m} / B_{N}\right)^{2} \leqq 2^{m}$. Furthermore

$$
r^{2 n_{N+m}}=r^{2 n_{N+1}} \frac{n_{N+m}}{n_{N+1}} \leqq(1 / 4)^{q^{m-1}} .
$$


Thus

Clearly,

$$
|Q| \leqq \epsilon_{N} \sum_{m=1}^{\infty} 2^{m}(1 / 4)^{q^{m-1}} \rightarrow 0
$$

$$
\begin{aligned}
\frac{S(r, x)}{\left(2 B_{r}^{2} \log \log B_{r}\right)^{1 / 2}}= & \frac{S_{N}(x)}{\left(2 B_{N}^{2} \log \log B_{N}\right)^{1 / 2}} \frac{\left(2 B_{N}^{2} \log \log B_{N}\right)^{1 / 2}}{\left(2 B_{r}^{2} \log \log B_{r}\right)^{1 / 2}} \\
& -\frac{S_{N}(x)-S(r, x)}{\left(2 B_{r}^{2} \log \log B_{r}\right)^{1 / 2}}
\end{aligned}
$$

Since

$$
\left(\frac{2 B_{N}^{2} \log \log B_{N}}{2 B_{r}^{2} \log \log B_{r}}\right)^{1 / 2} \rightarrow 1
$$

and

$$
\lim \sup \frac{S_{N}(x)}{\left(2 B_{N}^{2} \log \log B_{N}\right)^{1 / 2}}=1
$$

a.e., the first term on the right of (19.4) has lim sup equal to 1 a.e. and therefore it is sufficient to show

$$
\frac{S_{N}(x)-S(r, x)}{\left(2 B_{N}^{2} \log \log B_{N}\right)^{1 / 2}} \rightarrow 0
$$

for all $x$.

We will show

$$
\begin{gathered}
\frac{S_{N}(x)-S(r, x)}{B_{\mathrm{N}}} \rightarrow 0 . \\
\frac{S_{N}(x)-S(r, x)}{B_{N}} \leqq\left|\sum_{1}^{N} \frac{a_{k}}{B_{N}}\left(1-r^{n k}\right)\right|+\left|\sum_{m=1}^{\infty}\left(\frac{a_{N+m}}{B_{N+m}}\right)\left(\frac{B_{N+m}}{B_{N}}\right) r^{n k}\right| \\
\leqq \epsilon_{N} C \sum_{1}^{N}\left(\frac{1}{q}\right)^{N-k}+\epsilon_{N} \sum_{m=1} 2^{m}(1 / 2)^{q^{m}} \rightarrow 0 .
\end{gathered}
$$

20. It should be remarked that the Law of the Iterated Logarithm holds for lacunary power series.

Theorem 3. If

$$
S(x)=\sum_{k=1}^{\infty} c_{k} e^{i n_{k} x} ; \quad \frac{n_{k+1}}{n_{k}}>q>1
$$

has coefficients $c_{k}$ satisfying 
where

$$
\max _{1 \leqq k \leqq N}\left|c_{k}\right|=o\left\{\frac{B_{N}}{\left(\log \log B_{N}\right)^{1 / 2}}\right\}
$$

$$
B_{N}=\left(\sum_{k=1}^{N}\left|c_{k}\right|^{2}\right)^{1 / 2}
$$

then

$$
\lim \sup \frac{S_{N}(x)}{\left(B_{N}^{2} \log \log B_{N}\right)^{1 / 2}}=1
$$

almost everywhere. Furthermore, if

$$
B_{r}=\left(\sum_{k=1}^{\infty}\left|c_{k}\right|{ }^{2} r^{2 n_{k}}\right)^{1 / 2}
$$

then

$$
\lim \sup \frac{\left|S_{r}(x)\right|}{\left(B_{r}^{2} \log \log B_{r}\right)^{1 / 2}}=1
$$

almost everywhere.

It should be observed that there is no longer the factor $2^{1 / 2}$ in the denominator of (20.1).

As it has been remarked by Salem and Zygmund [1], the equation (20.1) for " $\leqq$ " instead of " $=$ " is an easy consequence of the theorem for trigonometric series. The opposite inequality is an even easier consequence of the trigonometric case as we can see by noting that

$$
\left|S_{N}(x)\right| \leqq\left|\Re S_{N}(x)\right|=\left|\sum_{1}^{N}\left(a_{k} \cos n_{k} x-b_{k} \sin n_{k} x\right)\right|
$$

where $c_{k}=a_{k}+i b_{k}$.

\section{REFERENCES}

P. ERDös ANd I. S. GÁL

1. On the law of the iterated logarithm, Nederl. Akad. Wetensch. Pruc. Ser. A. vol. 58 (1955) pp. $65-84$.

A. KolmogorofF

1. Über das Gesetz des Iterierten Logarithmus, Math. Ann. vol. 101 (1929) pp. 126-135. R. SAlem ANd A. Zygmund

1. La loi du logarithme itéré pour les séries trigonométriques lacunaires, Bull. Sci. Math. (2) vol. 74 (150) pp. 209-224.

A. ZYGmund

1. Trigonometrical Series, Warszawa-Lwow, Monografie Matematyczne, 1935.

University of Chicago,

Chicago, Ill. 